\title{
Interview with Rainer Hoffmann on "The Transformation Towards Artificial Intelligence of Electric Utilities"
}

\author{
Philipp Staudt
}

Published online: 29 March 2021

(c) Springer Fachmedien Wiesbaden GmbH, ein Teil von Springer Nature 2021

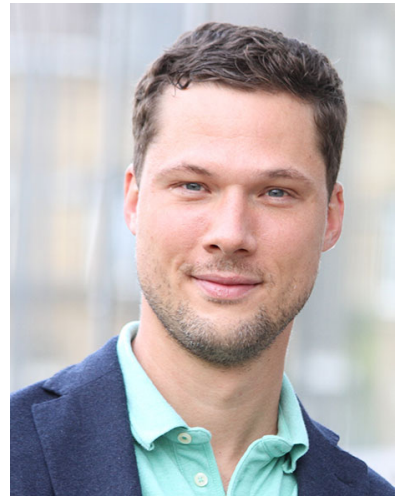

Dr. Rainer Hoffmann

EnBW AG

ra.hoffmann@enbw.com

Dr. Rainer Hoffmann is a Senior Manager in EnBW's Digital Transformation unit and leads its artificial intelligence activities. His work focuses on shaping the organization that enables scaling AI in the best way. He started his career as a data scientist and was later responsible for EnBW's algorithmic intraday trading activities. Rainer studied Industrial Engineering at the Karlsruhe Institute of Technology (KIT) and at the University of Toronto, Canada. He holds a Ph.D. in Operations Research from KIT.

BISE Hi Rainer, nice to talk to you and thanks for agreeing to participate in this interview! Could you briefly

P. Staudt $(\bowtie)$

Karlsruhe Institute of Technology, Kaiserstrasse 89,

76131 Karlsruhe, Germany

e-mail: philipp.staudt@kit.edu explain your role at the utility company EnBW and the role of your department?

Hoffmann I work in EnBW's digital transformation unit where our mission is to support all business units in their transformation toward more digitized processes and ideally toward implementing new digital business models. Within this department, I lead our Artificial Intelligence activities as applying $\mathrm{AI}$ is a transformational process as well. In this role, I enable colleagues to use machine learning to address their business problems. This includes identifying use cases, providing training to the colleagues who need it, bringing practitioners together and communicating about all AI related activities throughout the whole company.

BISE From your experience, what is the area where your colleagues within the business units need most support?

Hoffmann The most important ability is to actually identify potential use cases. After that, it is about finding the right algorithms but we hardly have issues with that. But these first steps - understanding what your problem is, that it can be solved with data, and how to get the data they really are the most challenging part. And this is where we put a lot of our effort in. We try to help our colleagues understand what AI actually is and what it can do for them. Many of them have heard of the term AI before, but our task is to demystify it. However, in the end, there is still a big difference between being a spectator sitting in the ranks and actually being part of the game yourself. And we want to get our colleagues to be able and confident to be part of the game. This whole transformation ought to be humancentered and our colleagues need to see how they can contribute. We can tell that our approach is successful: We recently hosted the EnBW AI week for which we had more than 500 registrations. This shows that the topic is rapidly moving forward to the center of our corporate culture. 
BISE Where would you say does EnBW stand in terms of its digital transformation and the use of AI tools?

Hoffmann In regard to our progress, we position ourselves by means of the applied AI maturity journey logic with four maturity levels. ${ }^{1}$ For everyone who is interested, I recommend reading the case study about our journey towards level $2 .^{2}$ On level 2, you are able to leverage AI in your company to actually create value. And then on level 3 you can actually scale your AI operations across the whole company. We are currently transitioning from 2 to 3 . For our team, this includes standardizing certain things like which cases we should pursue and what the right tools are to identify and address them. To this day, we have been working on more than 30 cases, and this feels like quite a number as these cases do not grow on trees and are not easily implemented. In the long run, we want to reach level 3 , which says that AI is scaled in all those areas where it is really useful. Level 4 is for companies whose entire business model is based on AI, which is probably not where our business model is moving within the next years.

BISE What has changed since you and your team started to promote AI in regards to how people interact with you?

Hoffmann The most perceivable change is that we have more and more ambassadors for AI within the business units. The people we started to educate really became multipliers so that we no longer need to help every department with identifying use cases. We have also organized this group of colleagues as a community so that they can share their knowledge and learn from each other. The big advantage of our ambassadors identifying cases is that they know the pain points of their business much better than we as a central unit do. The issues that they then identify cannot always be solved with AI, but our colleagues learn with every use case they evaluate. And once they have discovered a new use case, we help them translate their needs for the (data) engineers who can then design the solution. And finally, they can contribute to a greater acceptance of new solutions within their business units. Because ultimately, we might build great tools, but if no one uses them, we do not create any value. But our challenges still definitely remain more on the side of identifying the use cases and conceptualizing the solution than with implementing the solution.

BISE Would you say that there is a difference in the challenges of implementing AI in a utility company as compared to any other industrial company?

Hoffmann There are probably two small differences. First, the sheer importance of energy for society: As a utility company our task is to ensure that the energy supply

\footnotetext{
1 https://www.appliedai.de/journey.

${ }^{2}$ https://www.appliedai.de/maturity-level-2-case-study-enbw-ener gie-baden-w\% $3 \%$ BCrttemberg.
}

is steadily available. This means that certain solutions simply need to be very robust. And secondly, power is a very peculiar good both in terms of the engineering as well as the regulation side which requires an in-depth domain knowledge. But honestly, apart from that, I think that the difference to other industrial companies is not that big. Many industrial companies face similar challenges such as legacy IT systems, data silos, the need for more sensors and collection of their data, and generally a very heterogeneous landscape in terms of IT and databases. The notion that data actually has substantial value is new to most of them. And really, these issues remain the most substantial roadblocks for scaling AI. They are often more related to data engineering rather than to data science. This mindset that data is part of the DNA is probably reserved for the internet companies who started their business when all this tech was already available. But this also makes digitalizing a utility so interesting as cultural change is always part of it.

BISE What would you say are success factors for getting an AI strategy on the road in a rather traditional industry?

Hoffmann Personally, I think that it has a lot to do with company culture and how you frame the transition. For instance, we began by talking to different departments and they were all very curious right away because we gave them the prospect of utilizing AI for improving their business. I would generally say that if you are able to build on curiosity, you will probably be most successful. And in our case, this has worked very well. That said, we have not had a top-down transition. Instead of developing ideas centrally which would then be forced onto the business, we did a roadshow through a significant part of EnBW and gave an introduction to AI as well as encouraged our colleagues to find use cases where data might help them. It was rather a process of self-discovery for the departments where individuals who were curious, were trained on demand. And this gives you a commitment in different business units that you would not achieve through a topdown approach. I think if companies force a new technology like AI on their employees, they might fail since it appears like AI is done for AI's sake. The way new technologies are introduced in a company is probably part of its culture and hard to change.

BISE Is there still something that those organizations can do to facilitate an AI strategy?

Hoffmann It is important to create a mindset for AI at the management level. A common mistake is that managers consider AI projects as investments. They invest amount $\mathrm{x}$ and expect amount $\mathrm{y}$ as return on this investment. But AI projects are rather experiments or ventures than investments. It is impossible to tell in advance whether the project will be successful and what the outcome will be. And employees need to feel that they will not be held 
responsible if a good idea that was well executed turned out not to work in practice. This is the first step in getting people engaged for AI applications.

BISE Could you talk about a few lighthouse projects that you accompanied?

Hoffmann For example, we developed a solution for state estimation of transformers in distribution grids. On this voltage level, transparency is becoming more and more important. With this new approach, we are trying to forecast the state of transformers for the coming hours. This has to happen for about 2000 transformers simultaneously, so it needs to be sufficiently fast. Similarly, we developed forecasting tools for virtual power plants. For instance, if you install a new PV panel, we have little data to learn from for a forecast. However, we can often use and adapt data from similar assets to create an estimation. Further, we are currently operationalizing a predictive maintenance solution for the gas distribution infrastructure. You can see that the projects are quite diverse.

BISE If you look at your use cases, do you think that AI can help us with the transition to a more sustainable energy system?

Hoffmann In the end, AI is a technology. Whether it helps in achieving a specific objective depends very much on how you use it and therefore how you incentivize its use. In our case, we have set a corporate goal to be carbon neutral by 2035 , which is reflected in our strategy. Consequently, in our case, AI does drive sustainability. If we can use AI to operate offshore windfarms more efficiently, this makes operating them more attractive, thus supporting the energy transition. And we build offshore windfarms because it is part of our corporate strategy. However, as I pointed out, this does not happen automatically. It needs to be coupled with the will of becoming more sustainable and with clear executive support. For instance, I think that if we identified a case that has no real financial impact but greatly helps in reducing carbon emissions, then we would still do it because it supports our objective.

BISE Imagine you could ask the research community for help, what would you want them to do?

Hoffmann The beauty of working in science is the freedom of choosing whatever you want to work on. You are not bound by proving a business case or by having to be profitable. Therefore, I would want scientists to use this freedom to rethink how our industry works and thus address the relevant problems for our society. For instance, we probably know most of our operational issues, and they are so diverse across companies that I'm not sure that external suggestions would help us much at this point. But there are other things which my colleagues and I do not have the time and freedom to think about. For instance, could we completely change the energy supply chain and generate electricity more cheaply? Can we establish completely new business models within the energy sector? What are ways of reducing carbon emissions that do not create value but are not too costly either? As I said, emissions reduction is an important objective for us. But to date, it is probably not yet the key indicator. It's this thinking completely outside of the box that I would hope to get from the scientific community.

BISE Your daily work sounds like a task for an information systems student to me.

Hoffmann And it can be. This is certainly a role for people who are used to working at the interface between business and tech. These people need to be the face to the customer. Or rather the colleague in this case who then translates business needs to engineers. They are like translators who need to understand both sides and need to be able to communicate effectively. They also need to understand the challenges of both sides and develop the solutions with them. That's why soft skills like communication and empathy are much more important for this role than technical expertise. You need to have a human-centered mindset, you need to be enthusiastic about change and then ideally you are able to understand the bigger picture of what you are doing. I think that the essence of the issues we have talked about so far is that these innovations are only possible if they are driven by people who are enthusiastic about them. And we need facilitators who can foster this excitement.

BISE Thanks so much for the interview!

Hoffmann Sure, it was my pleasure! 